\title{
Reconstruction of congenital microtia after ear canaloplasty using V-Y advancement of a temporal triangular flap
}

\author{
Hae Yeon Park ${ }^{1 *}$, Kyeong-Tae Lee ${ }^{1 *}$, Eun-Ji Kim², Kap Sung $\mathrm{Oh}^{3}$ \\ ${ }^{1}$ Department of Plastic Surgery, Samsung Medical Center, Sungkyunkwan University School of Medicine, Seoul; ${ }^{2}$ Slow Plastic Surgery Clinic, \\ Jeju; ${ }^{3}$ Department of Plastic Surgery, Kangbuk Samsung Hospital, Sungkyunkwan University School of Medicine, Seoul, Korea
}

Background Reconstruction of congenital microtia remains challenging, particularly in patients with a history of ear canaloplasty due to insufficient regional soft tissue. The insertion of a tissue expander prior to implantation of the cartilage framework has traditionally been employed. However, this procedure could induce additional morbidity. Herein, we present a method using $\mathrm{V}-\mathrm{Y}$ advancement of a temporal triangular flap to gain additional soft tissue in these challenging cases.

Methods Congenital microtia patients with a history of ear canaloplasty who underwent auricular reconstruction using the Nagata technique between 2016 and 2020 were reviewed. To obtain additional soft tissue, V-Y advancement of a temporal triangular flap was performed concurrently with implantation of the costal cartilage framework, without prior insertion of a tissue expander. The outcomes of these patients with respect to postoperative complications and esthetics were evaluated.

Results Eight patients with bilateral lesions were included. No specific complications developed after the first-stage surgery. However, one patient experienced complications after the second stage (auricular elevation). An analysis of the esthetic results showed most patients had excellent outcomes, achieving a satisfactory convolution. The median number of operations needed to complete reconstruction was 2 , which was fewer than required using the conventional method with prior insertion of a tissue expander.

Conclusions In patients with a history of previous canaloplasty, V-Y advancement of a temporal triangular flap could serve as an alternative to tissue expansion for microtia reconstruction. This technique provided reliable and satisfactory results with a reduced number of surgical stages.

Keywords Congenital microtia / Surgical flaps / Tissue expansion
Correspondence: Kap Sung Oh Department of Plastic Surgery, Kangbuk Samsung Hospital,

Sungkyunkwan University School of Medicine, 29 Saemunan-ro, Jongnogu, Seoul 03181, Korea

Tel: +82-2-2001-2178

Fax: +82-2-2001-2177

E-mail: kapsung.oh@samsung.com

Received: March 4, 2021 • Revised: June 11, 2021 • Accepted: July 29, 2021

pISSN: 2234-6163・ elSSN: 2234-6171 • https://doi.org/10.5999/aps.2021.00381 • Arch Plast Surg 2021;48:614-621

\section{INTRODUCTION}

External ear reconstruction for congenital microtia remains challenging. Staged reconstruction using autologous costal car- tilage has been considered the gold standard for achieving reliable outcomes and low complication rates. The two-stage Nagata technique [1-4] is one of the most common methods. It involves implantation of a rib cartilage framework with lobule re-

Copyright $\odot 2021$ The Korean Society of Plastic and Reconstructive Surgeons

This is an Open Access article distributed under the terms of the Creative Commons Attribution Non-Commercial License (https://creativecommons.org/

licenses/by-nc/4.0/) which permits unrestricted non-commercial use, distribution, and reproduction in any medium, provided the original work is properly cited. I www.e-aps.org 
positioning in the first stage. Elevation of the reconstructed auricle occurs in the second stage.

During the first stage of the operation, a thin pliable skin envelope and an elaborately sculptured three-dimensional cartilage framework are required to achieve a satisfactory convoluted shape. When preparing the subcutaneous pocket for the cartilage framework, a sufficient skin envelope can easily be obtained using adjacent tissue. This includes remnant vestiges. However, in patients who have previously undergone surgical procedures such as canaloplasty at a location suitable for the cartilage framework graft, it is difficult to acquire a pliable skin envelope due to postoperative scar contracture or tissue deficiency.

Inserting the fabricated cartilage framework without resolving this issue can result in tightening of the overlying skin envelope. This can lead to unsatisfactory convolution or even necrosis. To avoid these problems and obtain additional soft tissue, a tissue expander is frequently inserted prior to the first stage of cartilage framework implantation [5]. However, this technique adds another surgical stage that requires general anesthesia. For unilateral microtia, this method requires at least three operations. Furthermore, to complete reconstruction for bilateral microtia, six operations are required. These multiple operations could represent a considerable burden for pediatric patients and their families, and they also pose a risk of foreign body-related complications, including infection.

A V-Y advancement flap is a traditional, simple procedure used to obtain additional soft tissue from surrounding areas. It has been used extensively for various types of reconstruction. For ear deformities, this method has played a key role in the correction of cryptotia [6-8]. However, it has not been used for the reconstruction of congenital microtia. We hypothesized that in patients with microtia and insufficient regional soft tissue, the V-Y advancement technique could yield an additional skin envelope. This technique is simple, reliable, and could provide satisfactory convolution.

\section{METHODS}

\section{Study population}

The Institutional Review Board of Samsung Medical Center (IRB No. 2020-05-121-002) approved this study. Using our prospectively maintained database, we searched for patients with congenital microtia who underwent external ear reconstruction using a rib cartilage graft with a modified Nagata technique between 2016 and 2020. Of these patients, we identified and reviewed: patients who had insufficient regional soft tissue due to a previous history of ear canaloplasty at the location of the cartilage framework graft; and patients who would be diffi- cult to treat using an ordinary technique. Preoperatively, the potential risks and benefits associated with the surgical methods were fully explained to the patients and their parents. Subsequently, they provided informed consent prior to surgery.

\section{Operative techniques}

All operations were performed by a single surgeon (KSO). V-Y flap advancement was performed concurrently with a rib cartilage graft. Fig. 1 shows a schematic drawing of the design of the temporal triangular flap for V-Y advancement. First, the location at which the cartilage framework was to be inserted was marked with consideration of the contralateral ear location. Next, a blue line was drawn beginning at the root of the helix and crossing the widest portion of the designed auricle. A temporal triangular flap was designed with an axis perpendicular to the blue line. The base width of the flap was the same as that of the blue line. The height was twice as long as the vertical distance from the peak of the helix to the blue line. The lower border of the flap terminated approximately $1 \mathrm{~cm}$ from the upper border of the designed helix. After an incision was made, the triangular flap was elevated from the temporal scalp above the superficial temporal fascia. Subcutaneous dissection proceeded inferiorly to the location at which the cartilage framework was to be implanted. Meticulous hemostasis kept the surgical field clear during pocket dissection. In cases lacking lobule tissue to be transposed, the subcutaneous pocket for framework implantation was prepared through a scalp incision without an additional incision. In cases with a small left lobule, another incision was made according to the Nagata

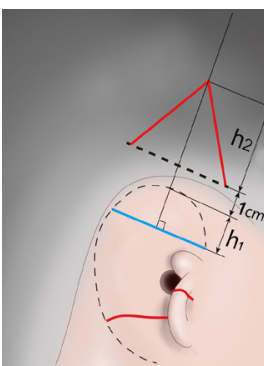

(A)

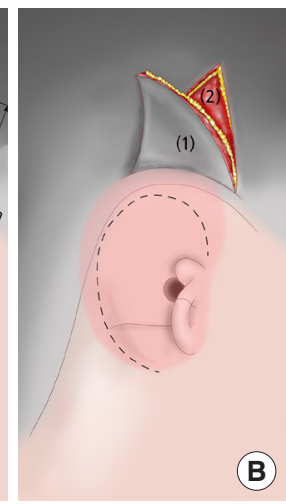

B

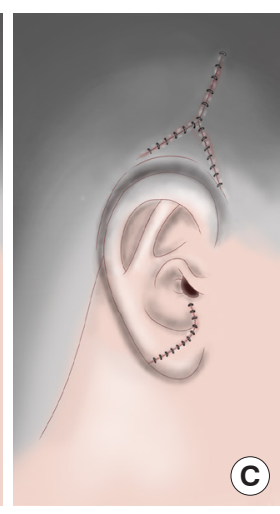

Fig. 1. Our V-Y advancement flap method. (A) Preoperative design. The location of the future reconstructed ear is depicted using a dotted line: temporal triangular flap (red line), the base width of the flap (blue line), incision line for lobule transposition (red, curvilinear line), vertical distance from the peak of helix to the blue line ( $h 1)$, and the height of the flap $\left(h_{2} ; h_{2}=2 \times h_{1}\right)$. (B) An elevated temporal triangular flap (1) is demonstrated, and the underlying superficial temporal fascia (2) is exposed. The shaded area represents the area of dissection for the framework insertion. (C) Immediate postoperative view. 
method. This enabled lobule transposition. Through the incision, subcutaneous pocket dissection was performed to ensure smooth insertion of the fabricated cartilage framework. After the fabricated cartilage framework was inserted, two negative suction drains were inserted. One drain was for the helical portion and the other was for the concha.

The triangular flap was advanced inferiorly to create an elastic skin envelope. Sufficient skin for the envelope was confirmed by verifying the convolution of the framework. This was performed while the skin flap was temporarily fixed with the application of negative-pressure suction. If the position of the cartilage framework was not situated as intended, it was repositioned after relieving the negative pressure. The cartilage framework could then be relocated until a perfect position was achieved. When ample skin was available, key sutures were inserted. For donor site closure, it was necessary to carefully undermine the adjacent temporal scalp to reduce skin tension. Special attention was paid to avoid injury to the underlying superficial temporal fascia and its vascular pedicle. A mild compressive dressing with fluffy gauze and an elastic bandage was applied. The first- and secondstage operative procedures were conducted as usual $[9,10]$.

\section{Data collection and outcome measures}

Patient-related information for variables including age, sex, type of microtia based on the Nagata classification, laterality of the affected ear, follow-up period, and history of canaloplasty was collected from the patients' medical records. Operation-related variables, including the date of surgery, postoperative complications, and surgical revisions, were also investigated. The outcomes of interest were postoperative complications and esthetic outcomes. Postoperative complications included those that developed during the reconstruction process. The esthetic outcomes were rated on a 4-point scale based on photographic data as follows: $1=$ poor, 2 =fair, $3=$ good, and $4=$ excellent. The evaluation processes were conducted separately for each surgical stage. First, the outcome of the rib cartilage graft was assessed using photographs taken prior to auricular elevation. Second, the outcome of auricular elevation was evaluated using photographs taken at the next visit after auricular elevation. Three plastic surgeons who were not involved in the operations independently reviewed the photographs of each patient and evaluated the convolution, ear size, and balanced projection.

\section{RESULTS}

\section{Baseline characteristics}

Eight patients with microtia who had a history of ear canaloplasty were treated during the study period. All patients had bi-
Table 1. Demographic characteristics of the study subjects

\begin{tabular}{lc}
\hline Characteristic & Value \\
\hline No. of cases & 8 \\
Age at canaloplasty (yr), average (range) & $5.6(1-10)$ \\
Age at rib cartilage grafting (yr), average (range) & $10.1(8-14)$ \\
Sex & \\
Male & 6 \\
Female & 2 \\
Type & \\
Lobule type & 8 \\
Concha type & 0 \\
Anotia & 0 \\
Laterality) & \\
Left & \\
$\quad$ Right & 4 \\
Follow-up period (mo), mean (range) & 4 \\
Time interval between the canaloplasty and the first stage & $52.8(11-104)$ \\
$\quad$ of auricular reconstruction (mo), mean (range) & \\
\hline
\end{tabular}

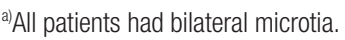

lateral microtia. The average age at the time of ear canaloplasty was 5.6 years (range, 1-10 years). The average age at the time of the first-stage operation was 10.1 years (range, $8-14$ years). The patient population comprised six males and two females. All patients were classified as having lobule-type microtia as defined by Nagata. The left ear was treated in four cases, and the right ear was treated in four cases. The mean follow-up period was 24.8 months, ranging from 17 months to 43 months (Table 1). The first stage of auricular reconstruction was performed at a mean time of 52.8 months (range, 11-104 months) after canaloplasty.

\section{Complications}

No complications occurred after the first stage of surgery. We observed mild skin discoloration or blanching at the framework implantation site immediately after the first stage of the operation. This was resolved within 2 weeks with conservative management (ointment was applied or the pressure of the dressing materials was relieved). After the second surgical stage, only one patient experienced complications. This patient had delayed healing with graft loss that required debridement and splitthickness skin grafting under general anesthesia. No patients experienced cartilage extrusion, destruction, resorption, or infection. In addition, there were no complications related to canaloplasty, such as chronic drainage of the auditory meatus and canal stenosis. Including revision surgery due to complications, the median number of operations conducted to complete reconstruction after ear canaloplasty was 2 .

\section{Aesthetic outcomes}

In the first assessment of the outcomes of the rib cartilage graft, 
Table 2. Aesthetic outcomes before and after auricular elevation

\begin{tabular}{|c|c|c|c|c|c|c|c|}
\hline \multirow[b]{2}{*}{ Group } & \multicolumn{3}{|c|}{ Aesthetic outcomes before auricular elevation } & \multicolumn{4}{|c|}{ Aesthetic outcomes after auricular elevation } \\
\hline & Convolution & Size & Final score ${ }^{a)}$ & Convolution & Size & $\begin{array}{l}\text { Balanced } \\
\text { projection }\end{array}$ & Final score ${ }^{a}$ \\
\hline Average score & 3.38 & 3.88 & 3.63 & 3.33 & 3.67 & 3.83 & 3.61 \\
\hline \multicolumn{8}{|c|}{ Specific distribution, No. (\%) } \\
\hline Excellent & $5(62.5)$ & $7(87.5)$ & & $3(50.0)$ & $4(66.7)$ & $5(83.3)$ & \\
\hline Good & $1(12.5)$ & $1(12.5)$ & & $2(33.3)$ & $2(33.3)$ & $1(16.7)$ & \\
\hline Fair & $2(25.0)$ & 0 & & $1(16.7)$ & 0 & 0 & \\
\hline Poor & 0 & 0 & & 0 & 0 & 0 & \\
\hline
\end{tabular}

${ }^{a}$ Mean values of the outcomes, rated using a 4-point scale (excellent=4, good=3, fair =2, poor $=1$ ).
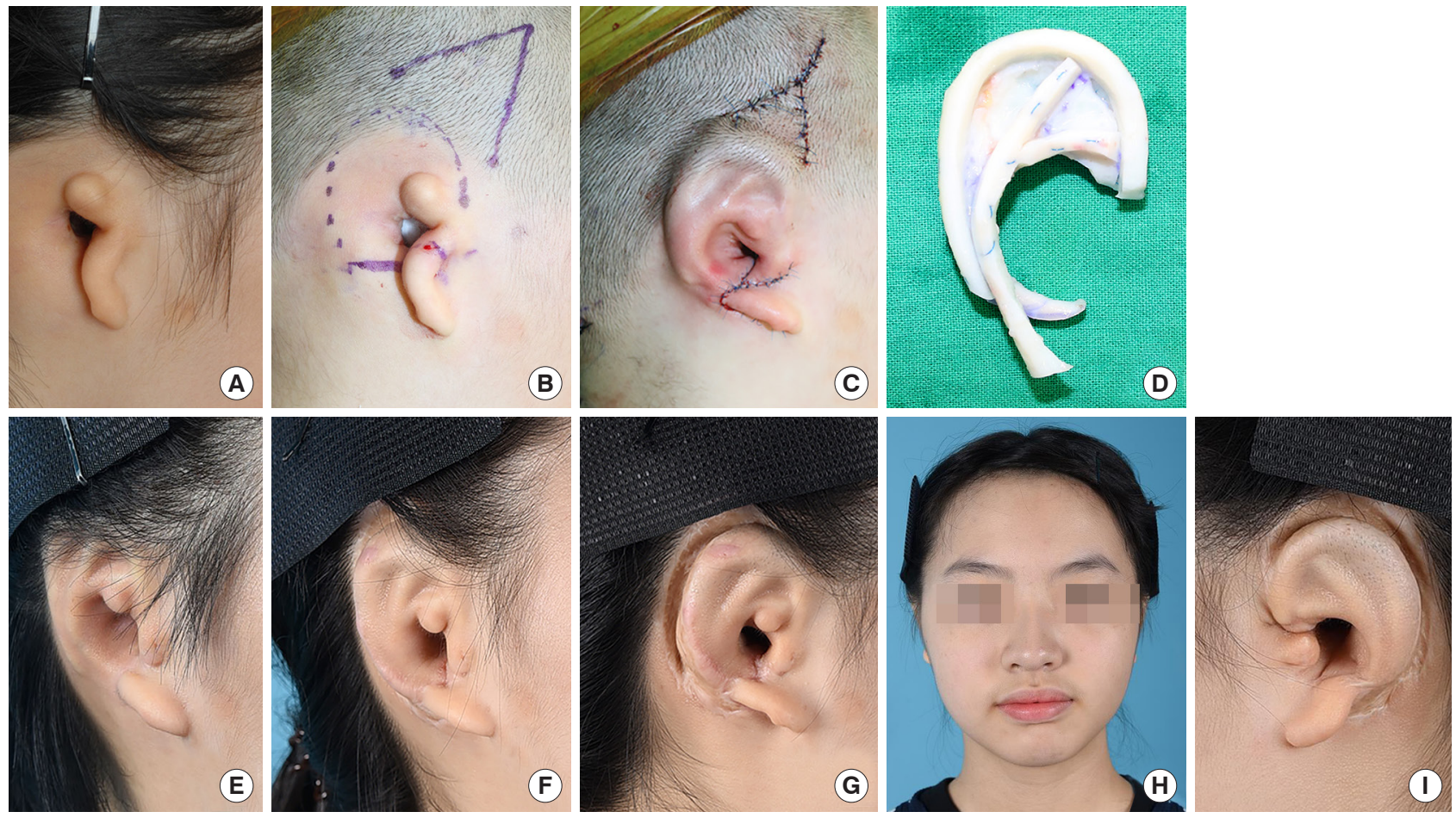

Fig. 2. Case 1. (A) Preoperative lateral view of a 9-year-old girl showing lobule-type microtia on the right side. (B) Preoperative design. (C) Immediate postoperative view. (D) Embedded three-dimensional cartilage framework. (E) Shape of the auricle 1 year after the autologous rib cartilage graft. (F-H) Projection of the auricle 1 year after elevation. (I) Lateral view of the reconstructed contralateral ear, which was constructed using the tissue expansion technique.

all study participants were evaluated. They received an average score of 3.38 with respect to convolution and 3.88 , with respect to size. When combining these scores into a single 4-point score, the average final score was 3.63. The second assessment of the outcomes of the final surgery included six patients. Two patients had not yet undergone auricular elevation. In the second assessment of the final operative outcomes, patients received an average score of 3.33 for convolution and 3.67 for size. Moreover, all patients achieved "excellent" and "good" outcomes in terms of balanced projection, with an average score of 3.83 . The average final score was 3.61 (Table 2). Three representative cases of auricular reconstruction using the V-Y advancement method are described below.

\section{Case reports}

\section{Case 1}

A 9-year-old girl presented with bilateral lobule-type microtia (Fig. 2). She underwent canaloplasty in both ears prior to auricular reconstruction. This induced a regional soft tissue deficiency. Five years after canaloplasty on the right ear, the right ear was reconstructed using a V-Y advancement flap with a concurrent rib cartilage graft in the first stage. Two years after rib cartilage grafting, the embedded framework was elevated for projection using a costal cartilage block. The temporoparietal fascia flap was elevated to cover the posterior cartilage graft, while the remaining defect was covered with a full-thickness skin graft from the groin. A natural appearance of the auricle was achieved and 

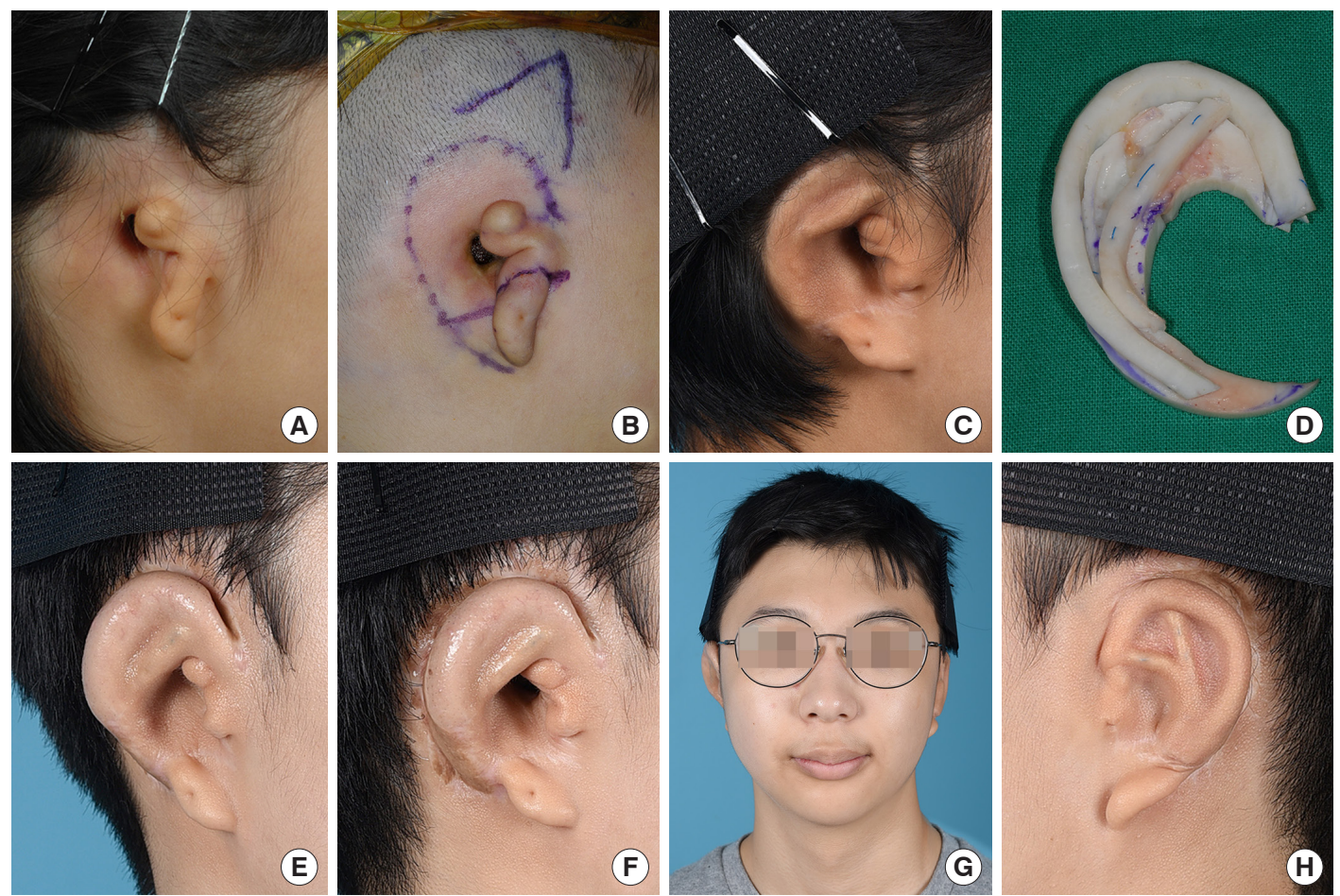

Fig. 3. Case 2. (A) Preoperative lateral view of a 10-year-old boy showing lobule-type microtia on the right side. (B) Preoperative design. (C) Shape of the auricle 2 years after autologous rib cartilage grafting. (D) Embedded three-dimensional cartilage framework. (E-G) Projection of the auricle 2 years after elevation. The patient had no problem wearing eyeglasses. $(H)$ Lateral view of the reconstructed contralateral ear, which was constructed using the modified Nagata technique. Canaloplasty was not performed on this side.
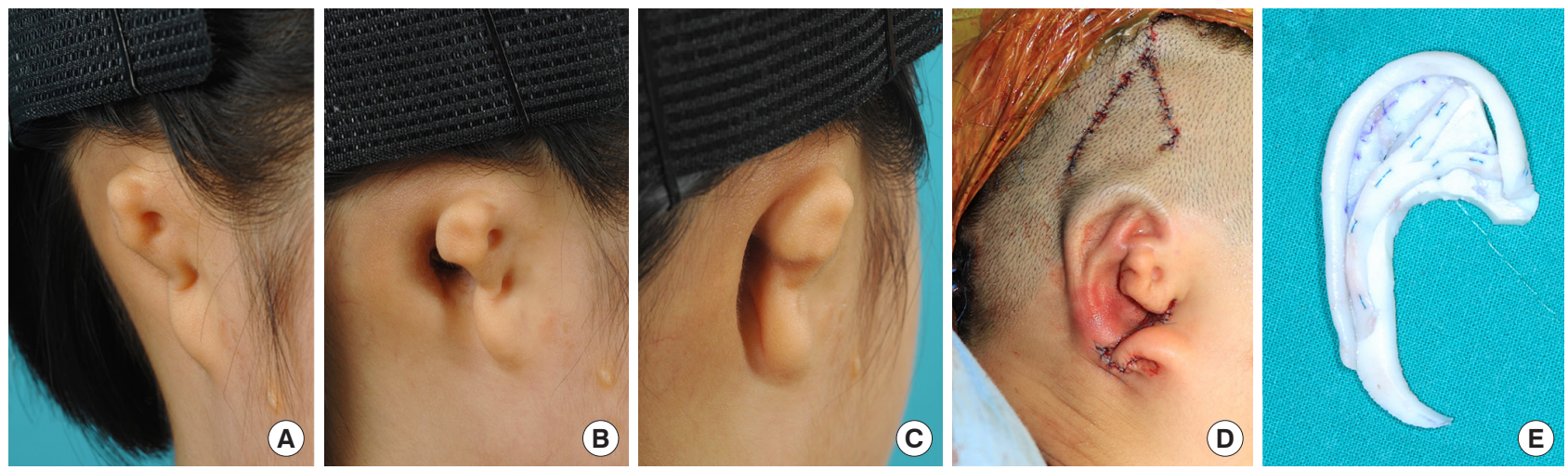

Fig. 4. Case 3. (A-C) Preoperative view of a 10-year-old boy showing lobule-type microtia on the right side. (D) Immediate postoperative view. (E) Embedded three-dimensional cartilage framework.

the esthetic outcome score was 4 at each assessment stage. In contrast to the right side, the left ear was reconstructed using the tissue expansion technique. This was conducted 2 years after canaloplasty.

\section{Case 2}

A 10-year-old boy presented with bilateral lobule-type microtia (Fig. 3). He underwent canaloplasty on only the right side 4 years before auricular reconstruction. The right ear was reconstructed using a V-Y advancement flap with a concurrent rib cartilage graft in the first stage. Two years after the rib cartilage grafting, auricular elevation was performed using a cartilage block and superficial temporal fascia. The remaining defect was covered with a full-thickness skin graft from the groin. The reconstructed ear appeared to be well-shaped and balanced. The esthetic outcome score was 4 at each assessment stage.

\section{Case 3}

A 10-year-old boy presented with bilateral lobule-type microtia (Fig. 4). He underwent canaloplasty on his right ear 4 years pri- 
or to auricular reconstruction. The ear was reconstructed using a V-Y advancement flap with a concurrent rib cartilage graft in the first stage. Immediate postoperative views showed good convolution despite soft tissue deficiency.

\section{DISCUSSION}

In patients with congenital microtia who have undergone previous canaloplasty, the traditional strategy for reconstruction has been to use tissue expansion to overcome the issue of regional soft tissue deficiency. However, this has the limitation of adding reconstruction stages and associated patient morbidity. There have been certain trials to replace this method with postauricular skin flaps. Kim [11] introduced a novel skin flap method using an extended scalp postauricular and isolated conchal flap. Hwang et al. [12] modified this method by extending the conchal flap and applied it to patients who had undergone previous canaloplasty. The results were satisfactory. However, this procedure required additional effort. Numerous elevated flaps were required as well as resurfacing with skin grafts from donor sites. In addition, there may be a potential risk of flap congestion, as multiple flaps converge on the embedded framework. In the current study, we adopted a simple method of V-Y advancement of a triangular temporal flap for reconstruction in eight microtia patients with challenging presentations. This method enabled us to obtain sufficient soft tissue, effectively reducing the number of operation stages and facilitating esthetically pleasing outcomes with a low complication rate.

As ear canal operations lead to vascular injury and scarring in the conchal region [7], many attempts have been made to minimize the undesired impacts of auricular reconstruction. This has been accomplished by adjusting the timing of each reconstruction stage. Roberson et al. [13] reported a higher meatal stenosis rate when atresia repair was conducted prior to Medpor insertion than when atresia repair was performed after microtia reconstruction. Chen et al. [14] introduced a new flap technique for combining canaloplasty simultaneously with total auricular reconstruction by performing Medpor framework insertion. This showed favorable cosmetic results with acceptable complication rates. At our institution, patients with unilateral microtia were routinely sent to otologists at the end of the first stage of hearing evaluation. We recommend canaloplasty prior to auricular elevation if the expected success rate of hearing restoration is over $90 \%$. Patients usually have functional hearing on the contralateral side [10]. However, for bilateral microtia patients, hearing restoration is a major concern that must be addressed to prevent delays in speech and language development [15]. Considering that we routinely use autogenous ribs for au- ricular construction, surgery should be postponed until rib growth provides sufficient cartilage for fabrication. Therefore, most of the patients underwent ear canaloplasty prior to auricular reconstruction. Thus, in this study, we focused on patients who had bilateral microtia with a history of canaloplasty prior to auricular reconstruction. We reviewed our experiences with microtia reconstruction using V-Y advancement of a temporal triangular flap in these cases and evaluated the outcomes.

Kubo [8] first reported a surgical method using a V-Y advancement flap for cryptotia correction. The V-Y advancement flap provided sufficient skin to the upper and posterior portions of the auricle, and was also easy to apply in almost all cases by regulating the height of the triangular flap [6]. In this study, we adopted a V-Y advancement technique for microtia reconstruction in cases with unfavorable regional conditions due to prior canaloplasty. Using this method, we achieved satisfactory results with respect to the costal cartilage graft and the auricular elevation. We also found that this method could be used safely without serious complications that could require revisional cartilage grafting. An imbalance between the skin envelope and the framework results in poor auricular definition. Moreover, a poorly vascularized skin envelope can result in skin necrosis, framework exposure, or long-term delayed cartilage resorption [16]. Considering these aspects, the use of the V-Y advancement flap could provide enhanced stability compared to the tissue expansion method. The V-Y advancement flap could keep its own vascularity with adequate flap thickness. In addition, our method can achieve direct visualization with only a $\mathrm{V}$ incision on the premise that sufficient dissection with meticulous hemostasis of the future implantation site and proper traction will be conducted. After the implantation of the cartilage framework, negative-pressure suction was applied while the skin flap was temporarily fixed. We could then ascertain whether the cartilage framework was in the proper position and whether the flap supplied ample skin. We could adjust the framework until we achieved desirable results, thereby guaranteeing the security of the embedded cartilage framework without an additional incision.

Compared to the tissue expansion technique, the V-Y advancement technique does not pose a risk for capsular contracture. Capsular contracture can lead to ambiguous definitions and can contribute to stenosis of the newly created external auditory canal (EAC). In addition, this technique is free of risk for foreign body-related infections, which can result in EAC stenosis. Furthermore, various strategies to prevent further EAC narrowing after auricular reconstruction have been adopted. We attempted to maintain the dermal and subdermal plexus. We also included a subcutaneous pedicle in the skin flap to ensure sufficient blood 
supply. A history of hematoma could result in EAC stenosis. Therefore, surgeons must be vigilant regarding bleeding. Careful hemostasis and proper drain placement must be conducted. If canaloplasty is planned after a rib cartilage graft, a wide conchal bowl is created at the framework fabrication stage. This provides space for future tissue contraction and canal stenosis [10]. A sufficient interval between operations (with an ideal time of at least 1 year) was also allowed to facilitate recovery. In addition, after ear canaloplasty, a customized ear mold was worn by the patient to prevent canal narrowing.

There are concerns regarding hair distribution when using the V-Y advancement flap method. When the triangular flap is inferiorly advanced, the hair-bearing portion of the flap base moves to the helical sulcus or possibly to the upper portion of the helix with a lower hairline. This can be successfully managed using laser epilation. We used a long-pulsed alexandrite laser system (GentleMax Pro, Candela Co., Wayland, MA, USA) under topical anesthesia with 5\% lidocaine cream. The irradiated region was within $2 \mathrm{~cm}$ of the outline of the cartilage framework implantation site, as determined by the amount of lowered hairline. The irradiation fluence was $18-22 \mathrm{~J} / \mathrm{cm}^{2}$ ( $3 \mathrm{~ms}$, spot size 12 $\mathrm{mm})$. In all cases, laser epilation was conducted after the rib cartilage graft. However, the optimal timing of laser epilation, either before or after auricular elevation, depends on the patient's needs. We performed irradiation at least 2 months after the previous operation. The irradiation interval was every 4-6 months. If patients were satisfied with their appearance without additional irradiation, we completed the epilation procedures and encouraged patients to visit the clinic as needed. There were no side effects such as inflammation, scarring, skin contracture, or circulatory failure related to irradiation. With respect to donor site morbidity, a Y-shaped scar was inevitable. However, the scar can be hidden by hair growth.

Although our method using temporal V-Y advancement could provide sufficient soft tissue in the first surgical stage, and the rib cartilage graft demonstrated excellent convolution, concerns persist regarding the perfusion of the cartilage framework implantation site or the advanced triangular flap. In the first stage of the operation, we designed the lower border of the temporal triangular flap $1 \mathrm{~cm}$ from the designed helix. By distinguishing the flap from the cartilage framework, more perforators would be included in the flap. It also protects flap from the threat of ischemia. In the presented cases, we observed temporary ischemic signs on the framework implantation site immediately after rib cartilage implantation. However, these signs resolved quickly with conservative management. In the second stage of the operation, the transposed flap, the margin of which was surrounded by scar tissue, was elevated to harvest the superficial temporal fascia. The flap potentially could have had insufficient tissue perfusion, which could have led to necrosis. However, in the present study, the majority of patients had an uneventful course after the second surgical stage. Only one patient had partial necrosis of the transposed triangular flap. It is likely that, with its elaborate design that considered the incision line of the second surgical stage as well as the meticulous technique involved, our method would not result in adverse outcomes following the second surgical stage.

The method described in this study exhibited satisfactory esthetic outcomes in all patients and produced stable reconstructive outcomes. However, as we included only a small number of cases, the final outcomes of this technique require confirmation through further clinical research.

\section{NOTES}

\section{Conflict of interest}

No potential conflict of interest relevant to this article was reported.

\section{Ethical approval}

The study was approved by the Institutional Review Board of Samsung Medical Center (IRB No. 2020-05-121-002) and performed in accordance with the principles of the Declaration of Helsinki. Written informed consent was obtained.

\section{Patient consent}

The patients provided written informed consent for the publication and the use of their images.

\section{Author contribution}

Conceptualization: KS Oh, KT Lee. Data curation: HY Park, EJ Kim. Formal analysis: KT Lee, HY Park. Methodology: KS Oh, KT Lee. Project administration: KS Oh. Visualization: HY Park, EJ Kim. Writing - original draft: KT Lee, HY Park. Writing - review \& editing: KS Oh, KT Lee. Approval of final manuscript: all authors

\section{ORCID}

Hae Yeon Park https://orcid.org/0000-0002-2872-0590

Kyeong-Tae Lee https://orcid.org/0000-0002-9070-9296

Eun-Ji Kim

Kap Sung Oh

https://orcid.org/0000-0002-3864-822X

https://orcid.org/0000-0002-5535-8400

\section{REFERENCES}

1. Nagata S. Modification of the stages in total reconstruction 
of the auricle: part I. Grafting the three-dimensional costal cartilage framework for lobule-type microtia. Plast Reconstr Surg 1994;93:221-30.

2. Nagata S. Modification of the stages in total reconstruction of the auricle: part II. Grafting the three-dimensional costal cartilage framework for concha-type microtia. Plast Reconstr Surg 1994;93:231-42.

3. Nagata S. Modification of the stages in total reconstruction of the auricle: part III. Grafting the three-dimensional costal cartilage framework for small concha-type microtia. Plast Reconstr Surg 1994;93:243-53.

4. Nagata S. Modification of the stages in total reconstruction of the auricle: part IV. Ear elevation for the constructed auricle. Plast Reconstr Surg 1994;93:254-66.

5. Park C, Mun HY. Use of an expanded temporoparietal fascial flap technique for total auricular reconstruction. Plast Reconstr Surg 2006;118:374-82.

6. Cho YK, Bae SG, Cho BC. Comparison between Z-plasty and $\mathrm{V}-\mathrm{Y}$ advancement for the surgical correction of cryptotia. Arch Craniofac Surg 2014;15:7-13.

7. Cho BC, Han KH. Surgical correction of cryptotia with V-Y advancement of a temporal triangular flap. Plast Reconstr Surg 2005;115:1570-81.

8. Kubo I. Taschenohr und Otoplastik. Oto-Rhino-Laryngologie 1933;6:105-10.

9. Lee KT, Oh KS. Predictors for unfavorable projection of the constructed auricle following ear elevation surgery in micro- tia reconstruction. Plast Reconstr Surg 2018;141:993-1001.

10. Kim A, Lee H, Oh KS. Review of 602 microtia reconstructions: revisions and specific recommendations for each subtype. Plast Reconstr Surg 2020;146:133-42.

11. Kim YS. A new skin flap method for total auricular reconstruction: extended scalp skin flap in continuity with postauricular skin flap and isolated conchal flap. Four skin flaps and temporoparietal fascia flap. Ann Plast Surg 2011;67: 367-71.

12. Hwang E, Kim YS, Chung S. A new skin flap method for total auricular reconstruction in microtia patients with a reconstructed ear canal: extended scalp and extended mastoid postauricular skin flaps. J Plast Reconstr Aesthet Surg 2014; 67:770-4.

13. Roberson JB Jr, Reinisch J, Colen TY, et al. Atresia repair before microtia reconstruction: comparison of early with standard surgical timing. Otol Neurotol 2009;30:771-6.

14. Chen $\mathrm{K}$, Jiang $\mathrm{C}, \mathrm{Wu} \mathrm{Q}$, et al. A new flap technique for reconstruction of microtia and congenital aural atresia. Indian J Surg 2015;77(Suppl 3):1237-41.

15. Chang SO, Lee JH, Choi BY, et al. Long term results of postoperative canal stenosis in congenital aural atresia surgery. Acta Otolaryngol Suppl 2007; (558):15-21.

16. Park JY, Park C. Microtia reconstruction in hemifacial microsomia patients: three framework coverage techniques. Plast Reconstr Surg 2018;142:1558-70. 